\title{
ARTICLE
}

\section{Response evaluation of Onion-like single Bonner sphere neutron spectrometer using TRUST Eu:LiCAF scintillator}

\author{
Kenichi Watanabe $^{\mathrm{a}^{*}}$, Tomoaki Mizukoshi ${ }^{\mathrm{a}}$, Atsushi Yamazaki ${ }^{\mathrm{a}}$, Sachiko Yoshihashi $^{\mathrm{a}}$, Akira Uritani ${ }^{\mathrm{a}}$, Tetsuo Iguchi ${ }^{\mathrm{a}}$, \\ Tomohiro Ogata $^{\mathrm{b}}$, Takashi Muramatsu ${ }^{\mathrm{b}}$, Tetsuro Matsumoto ${ }^{\mathrm{c}}$ and Akihiko Masuda ${ }^{\mathrm{c}}$ \\ ${ }^{a}$ Nagoya University, Furo-cho, Chikusa-ku, Nagoya, Aichi, 464-8603, Japan; ${ }^{b}$ Mitsubishi Heavy Industries Ltd., 1-1-1 \\ Wadamisaki-cho, Hyogo-ku, Kobe, Hyogo, 652-8585, Japan; ' National Institute of Advanced Industrial Science and Technology, \\ 1-1-1 Umezono, Tsukuba, Ibaraki, 305-8568, Japan
}

\begin{abstract}
We have developed the single sphere neutron spectrometer with five sensitive layers using the TRUST Eu:LiCAF scintillator and the WLSF readout. We evaluated the energy dependence of the detector response at thermal neutron and $24 \mathrm{keV}, 144 \mathrm{keV}, 565 \mathrm{keV}, 5 \mathrm{MeV}$ and $14.8 \mathrm{MeV}$ mono-energetic neutron irradiation fields. The experimentally determined neutron sensitivities are consistent with the Monte Carlo calculations. The detector responses for a bare and a heavy-water moderated ${ }^{252} \mathrm{Cf}$ sources also show good agreement with the expected ones using the Monte Carlo calculations.
\end{abstract}

Keywords: neutron detection; Bonner sphere neutron spectrometer; TRUST Eu:LiCAF scintillator; response function; standard neutron irradiation field

\section{Introduction}

Neutron spectrometers are widely used in nuclear facilities, space applications and medical applications. Neutron energy spectrum is required to determine neutron dose and also to estimate neutron activation amount in facility structural materials. Estimation of the neutron activation amount is quite important when decommissioning facilities. Recently, information of neutron flux and spectrum can be calculated precisely with Monte Carlo simulation codes, such as MCNP and PHITS [1,2]. However, calculations in complicated and large geometry are still difficult to get sufficient statistics or high precision. Measurements of neutron spectra, therefore, are essentially required. Additionally, spectrum measurements are also important to validate calculation results.

A Bonner sphere spectrometer (BSS) is widely used as representative of neutron spectrometers [3]. The BSS consists of several neutron detectors with different energy responses, which are thermal neutron detectors such as ${ }^{3} \mathrm{He}$ and $\mathrm{BF}_{3}$ detectors surrounded with polyethylene moderator sphere. Detector outputs or neutron counts obtained from the various size Bonner spheres are used to determine energy spectrum with the unfolding or spectrum adjustment technique.

The conventional BSS should take several measurements with the different detectors at an interest measurement point. In order to complete a measurement

*Corresponding author. Email: k-watanabe@energy.nagoya-u.ac.jp without changing detectors, development of a neutron spectrometer using a single detector, which has several outputs with different energy responses, has been desired.

Our research group developed novel neutron detectors consisting of a spherical moderator and positon sensitive thermal neutron detection elements, such as position sensitive ${ }^{3} \mathrm{He}$ tubes or discretely positioned $\mathrm{Li}$ glass scintillators [4,5]. These detectors can obtain spatial distribution of thermal neutrons in the moderator. The thermal neutron spatial distribution reflects energy spectrum of incident neutrons. This kind of detectors can provide information equivalent to the BSS. However, the response of these detectors has directional dependence due to geometric anisotropy in spatial arrangement of detection elements.

We, therefore, proposed and fabricated the neutron detector consisting of a single spherical moderator and multiple neutron sensitive shell layers with an onion-like structure [6]. The fabricated spectrometer has five sensitive layers. As a thermal neutron sensitive element, a transparent rubber sheet type Eu:LiCaAlF 6 (TRUST Eu:LiCAF) scintillator was applied [7]. In this transparent rubber sheet, a large number of small Eu:LiCAF pieces are dispersed with adequate spacing between them in order to suppress signal pulse height induced by a fast electron generated from a gamma-ray interaction. These fast electrons easily escape out of the small scintillators before fully depositing its energy. Several TRUST Eu:LiCAF scintillator bars were isotopically arranged in each sensitive layer. This type 
of scintillator is easy to be arranged in spherical layers due to its flexibility. The TRUST Eu:LiCAF scintillator can also apply the wavelength-shifting fiber (WLSF) readout, which is usually used for scintillation signal readout from large area scintillators [8]. We already evaluated the isotropic response of the fabricated onion-like single Bonner sphere [6]. In this paper, we evaluate the energy response of the fabricated detector.

\section{Onion-like single Bonner sphere}

The fabricated onion-like single Bonner sphere has five sensitive layers. Figure 1 shows the conceptual drawing of the detector. The outer diameter of the sphere is $216 \mathrm{~mm}$. The moderator sphere is made of polyethylene. The thickness of each moderator layer is 5 , $15,15,50 \mathrm{~mm}$ from the outer shell. Four flexible TRUST Eu:LiCAF scintillator bands with the WLSF readout are wrapped on each shell with equal angular intervals. The width of the scintillator bands is $5 \mathrm{~mm}$. Each scintillator band is wrapped with Teflon tape as a light reflector. The both ends of WLSFs are bundled for each layer and connected to five photomultiplier tubes (PMTs). The total weight of the detector is $6 \mathrm{~kg}$. The whole detector is encapsulated with aluminum housing for shielding ambient light. The signals from the PMTs are fed into a digital multichannel analyzer system to obtain signal pulse height spectra. Each layer is numbered from first to fifth layer from the innermost one. The outermost shell, therefore, is the fifth layer.

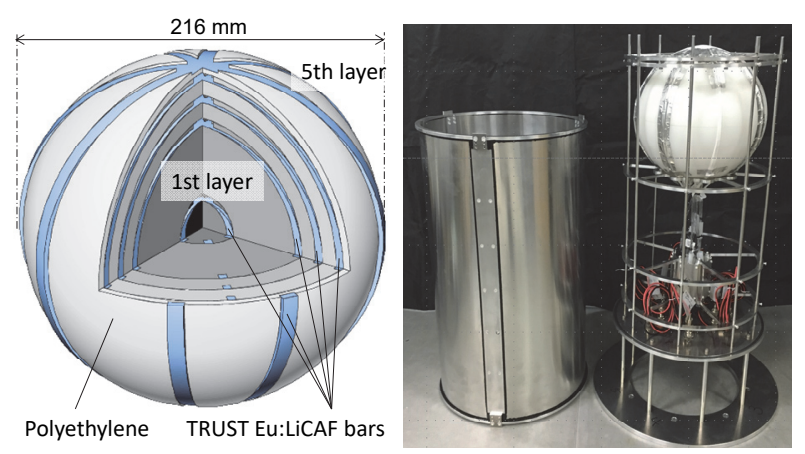

Figure 1. Conceptual drawing and photograph of the fabricated onion-like single Bonner sphere detector.

\section{Experiments}

We evaluated the energy response of the fabricated detector. The response evaluation tests were conducted at national standard neutron irradiation field in National Metrology Institute of Japan (NMIJ) of National Institute of Advanced Industrial Science and Technology (AIST), Japan [9]. The detector was irradiated with thermal neutrons and $24 \mathrm{keV}, 144 \mathrm{keV}, 565 \mathrm{keV}, 5 \mathrm{MeV}$ and $14.8 \mathrm{MeV}$ mono-energetic neutrons. In addition, we measured the responses under irradiation with a bare ${ }^{252} \mathrm{Cf}$ source and a heavy-water moderated ${ }^{252} \mathrm{Cf}$ source for validation purposes.

Thermal neutrons were produced with ${ }^{241} \mathrm{Am}-\mathrm{Be}$ neutron source placed at the center of a graphite pile with the dimension of $2,300 \times 1,900 \times 1,900 \mathrm{~mm}^{3}$. 24 $\mathrm{keV}, 144 \mathrm{keV}$ and $565 \mathrm{keV}$ mono-energetic neutrons were produced by ${ }^{7} \mathrm{Li}(\mathrm{p}, \mathrm{n}){ }^{7} \mathrm{Be}$ reactions using a proton beam from a $4 \mathrm{MV}$ Pelletron accelerator. For $24 \mathrm{keV}$ neutron production, an iron filter was additionally used to improve spectrum purity. $5 \mathrm{MeV}$ neutrons were produced with DD reactions using a deuteron beam from the $4 \mathrm{MV}$ Pelletron. 14.8 MeV neutrons were produced with DT reactions using also a deuteron beam from a $300 \mathrm{kV}$ Cockcroft-Walton accelerator.

Neutron sources are placed on an aluminum-grating floor supported at the center and mid-height of the relatively large irradiation room to reduce scattered neutrons from a floor and walls. We, additionally, applied the shadow-cone technique to subtract the background due to neutrons scattered in the irradiation room. Each measurement was made several times under the same condition to confirm reproduction of data.

\section{Results and discussion}

Figure 2 shows an example of the pulse height spectrum obtained from the fabricated detector. We can see a clear peak shape in the spectrum in spite of a complicated shape of the neutron sensitive scintillator and the use of the WLSF readout, in which the light collection efficiency is relatively low. This feature can allow us to re-adjust the discrimination level during a measurement while monitoring the spectrum. This is one of the advantages of applying the TRUST Eu:LiCAF and the WLSF readout. In this paper, the discrimination level was set at the center of the peak to remove gamma-ray events. Lower pulse height signals than the peak center might include gamma-ray induced events.

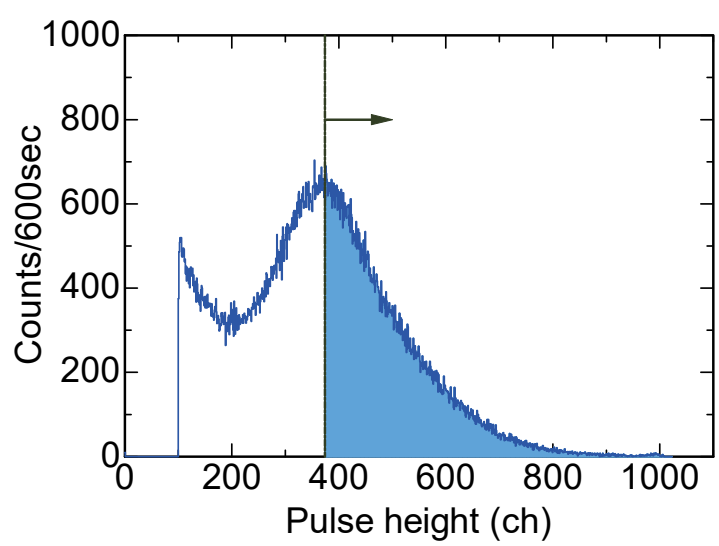

Figure 2. Example of the pulse height spectrum obtained from the fabricated detector. The data were taken from the fourth layer from the most inner shell when irradiating 144 $\mathrm{keV}$ neutrons.

The neutron sensitivity was evaluated as the detector response. The sensitivity is defined as the neutron counts per unit fluence. The net neutron counts were determined by subtracting the counts with the shadow cone from ones without it. The evaluated neutron 
sensitivities were compared with Monte Carlo simulation results using the PHITS code [2]. In the Monte Carlo calculations, the number of ${ }^{6} \mathrm{Li}(\mathrm{n}, \mathrm{t}) \alpha$ reactions in the Eu:LiCAF scintillator can be determined. As described above, we counted only half of neutron events occurred in the scintillator. In addition, the wall effect, in which charged particles generated in neutron induced reactions can significantly escape out of the scintillator, takes place in the TRUST Eu:LiCAF scintillator. This means that some fraction of neutron induced events migrates from the peak region to the lower side. The loss fraction due to the wall effect in the TRUST Eu:LiCAF is roughly $20 \%$. Consequently, the signals exceeding the discrimination level or the peak center is roughly $40 \%$ of the neutron induced events. This fraction is counting efficiency for neutron events. The counting efficiency is not precisely $40 \%$ but slightly depends on each individual detector element. We estimated the counting efficiency of each sensitive layer by comparing the experimentally determined sensitivity and the Monte Carlo calculation results. The counting efficiencies of each layer are listed in Table 1. These values are consistent with the above discussion. Figure 3 shows the energy dependence of the experimentally determined detector sensitivities of each sensitive layer. The Monte Carlo calculation results, which are corrected with the counting efficiency, are also plotted. The experimentally determined sensitivities agree well with the Monte Carlo calculation results. The detector of the third layer had relatively large difference to the calculated one because it was unstable in these measurements. This validation process can allow us to use the detector response determined by the Monte Carlo calculations for the unfolding procedure.

Table 1. The counting efficiency of each sensitive layer.

\begin{tabular}{cc} 
Layer & Correction factor \\
\hline 1st (the most inner) & 0.37 \\
2nd & 0.32 \\
3rd & 0.32 \\
4th & 0.36 \\
5th (The most outer) & 0.33
\end{tabular}

For validation purposes, the detector responses experimentally obtained from the fabricated detector in more practical cases are compared with ones expected from the already evaluated neutron spectra using Monte Carlo calculations. Figure $\mathbf{4}$ shows the detector responses when irradiating it with a bare ${ }^{252} \mathrm{Cf}$ and a $\mathrm{D}_{2} \mathrm{O}$ moderated ${ }^{252} \mathrm{Cf}$ sources. We can see that the experimentally obtained response is in good agreement with the expected one. The outer sensitive layers showed higher response to the $\mathrm{D}_{2} \mathrm{O}$ moderated ${ }^{252} \mathrm{Cf}$ source than the bare one because slow neutrons preferably interact with the outer layers. This result is reasonable considering the detector feature.

\section{Conclusions}

We evaluated the response of the onion-like single BSS using the TRUST Eu:LiCAF scintillator and the WLSF readout. The energy dependence of the detector a) 1st layer(the most inner)

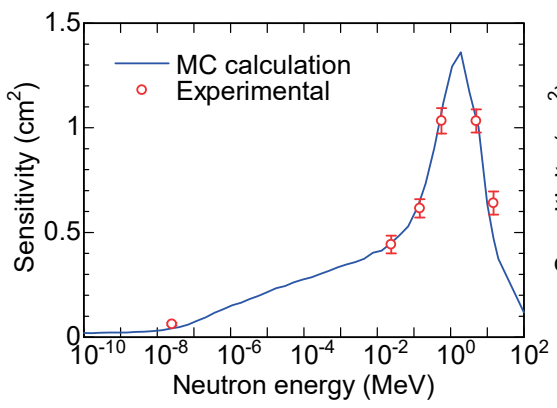

d) 4th layer

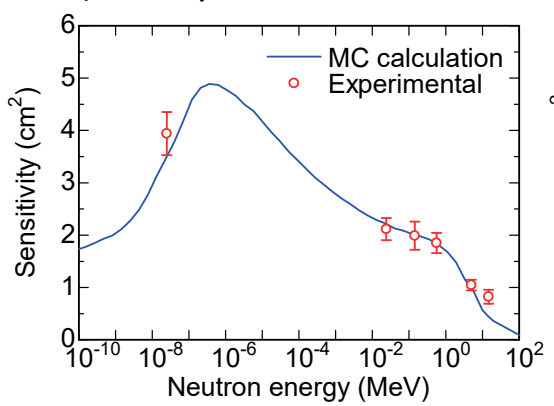

b) 2nd layer

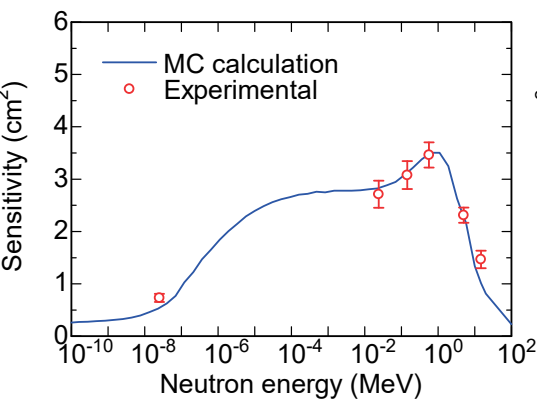

e) 5th layer(the most outer)

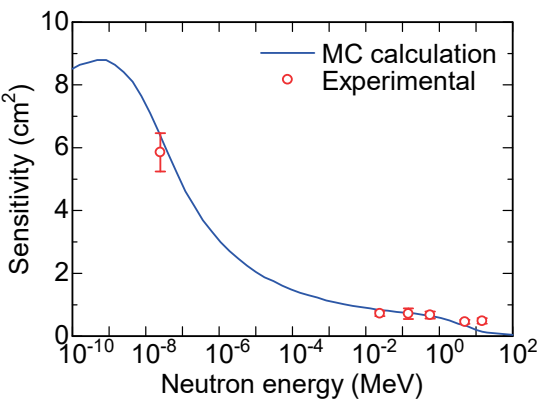

c) 3rd layer

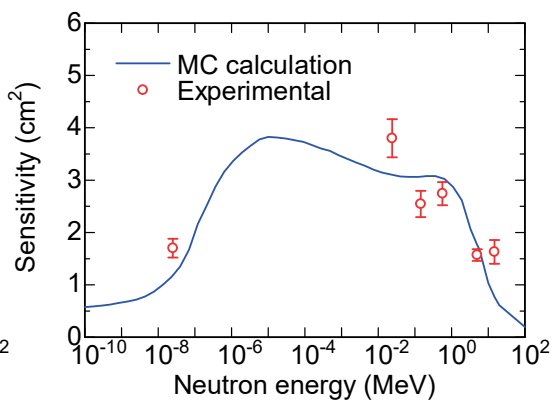

Figure 3. Energy dependence of the experimentally determined detector sensitivities of each sensitive layer (open circles). The Monte Carlo calculation results are also plotted (solid lines). The calculated responses are corrected by taking into account of the counting efficiency. 
a) Cf spectrum

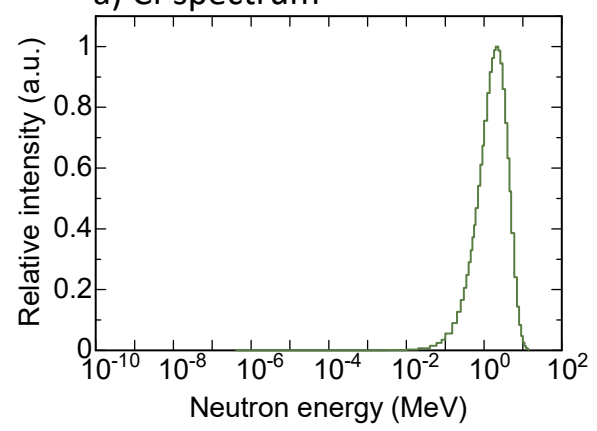

c) $\mathrm{D}_{2} \mathrm{O}$-Cf spectrum

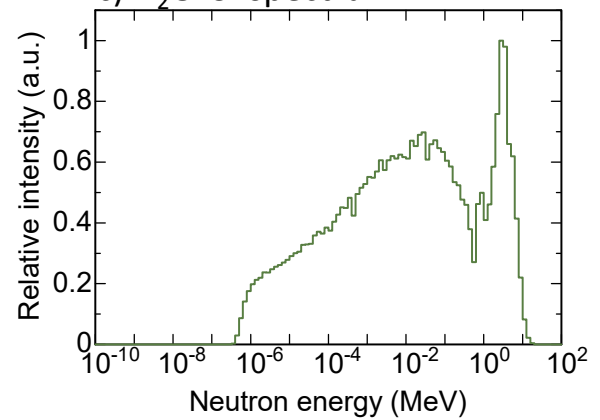

b) Cf response

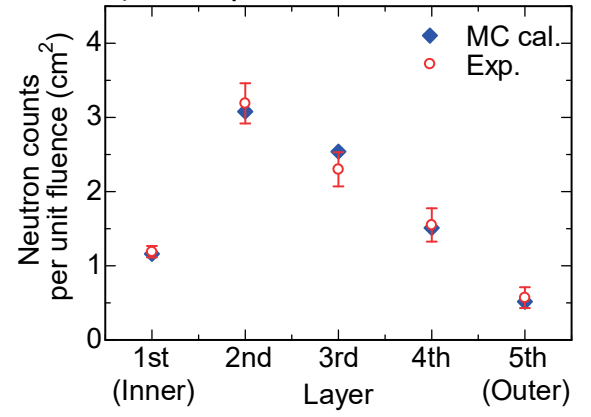

d) $\mathrm{D}_{2} \mathrm{O}$-Cf response

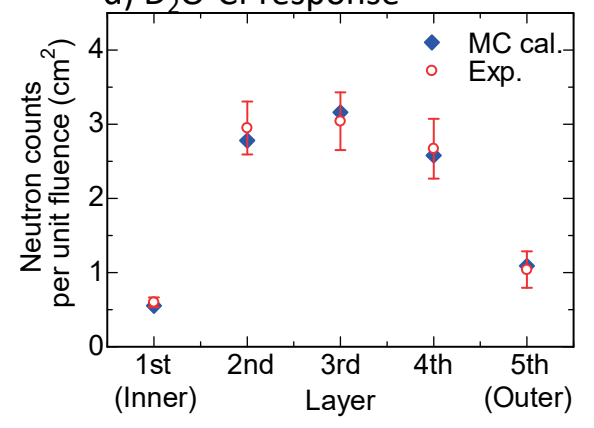

Figure 4. Detector responses irradiated with a bare ${ }^{252} \mathrm{Cf}$ and a $\mathrm{D}_{2} \mathrm{O}$ moderated ${ }^{252} \mathrm{Cf}$ sources. a) a bare ${ }^{252} \mathrm{Cf}$ source spectrum, b) detector response for a bare ${ }^{252} \mathrm{Cf}$, c) a $\mathrm{D}_{2} \mathrm{O}$ moderated ${ }^{252} \mathrm{Cf}$ source spectrum, d) detector response for a $\mathrm{D}_{2} \mathrm{O}$ moderated ${ }^{252} \mathrm{Cf}$. The experimentally obtained responses are plotted with the expected values by the Monte Carlo calculations.

response was measured by irradiating it with several energies of mono-energetic neutrons. The experimentally determined response well agrees with the Monte Carlo calculations. The neutron counts when irradiating it with a bare and $\mathrm{D}_{2} \mathrm{O}$ moderated ${ }^{252} \mathrm{Cf}$ sources are also in good agreement with the expected ones using the Monte Carlo calculations.

\section{References}

[1] T. Goorley, M. James, T. Booth, F. Brown, J. Bull, L. J. Cox, J. Durkee, J. Elson, M. Fensin, R. A. Forster, J. Hendricks, H. G. Hughes, R. Johns, B. Kiedrowski, R. Martz, S. Mashnik, G. McKinney, D. Pelowitz, R. Prael, J. Sweezy, L. Waters, T. Wilcox and T. Zukaitis, Initial MCNP6 release overview, Nucl. Technol. 180 (2012), pp. 298-315.

[2] T. Sato, K. Niita, N. Matsuda, S. Hashimoto, Y. Iwamoto, S. Noda, T. Ogawa, H. Iwase, H. Nakashima, T. Fukahori, K. Okumura, T. Kai, S. Chiba, T. Furuta and L. Sihver, Particle and heavy ion transport code system PHITS, version 2.52, J. Nucl. Sci. Technol. 50 (2013), pp. 913-923.

[3] R.L. Bramblentt, R.I. Ewing and T.W. Bonner, A new type of neutron spectrometer, Nucl. Instrum. Methods, 9 (1960), pp. 1-12.

[4] H. Toyokawa, A. Uritani, C. Mori, M. Yoshizawa, N. Takeda and K. Kudo, Neutron spectrometer with position-sensitive proportional counters, $\mathrm{Nucl}$. Instrum. Methods Phys. Res. A 381 (1996), pp. 481-487.
[5] S. Yamaguchi, A. Uritani, H. Sakai, C. Mori, T. Iguchi, H. Toyokawa, N. Takeda and K. Kudo, Spherical neutron detector for space neutron measurement, Nucl. Instrum. Methods Phys. Res. A 422 (1999), pp. 600-605.

[6] T. Mizukoshi, K. Watanabe, A. Yamazaki, A. Uritani, T. Iguchi and T. Ogata, T. Muramatsu, Upgrade of neutron energy spectrometer with single multilayer bonner sphere using onion-like structure, J. Rad. Protec. Res. 41 (2016), pp. 185-190.

[7] D. Sugimoto, K. Watanabe, K. Hirota, A. Yamazaki, A. Uritani, T. Iguchi, K. Fukuda, S. Ishidu, N. Kawaguchi, T. Yanagida, Y. Fujimoto, A. Yoshikawa, H. Hasemi, K. Kino and Y. Kiyanagi, Neutron TOF experiments using transparent rubber sheet type neutron detector with dispersed small pieces of $\mathrm{LiCaAlF}_{6}$ scintillator, Physics Procedia 60 (2014), pp. 349-355.

[8] K. Watanabe, T. Yamazaki, D. Sugimoto, A. Yamazaki and A. Uritani, T. Iguchi, K. Fukuda, T. Yanagida, Y. Fujimoto, Wavelength-shifting fiber signal readout from Transparent RUbber SheeT (TRUST) type $\mathrm{LiCaAlF}_{6}$ neutron scintillator, $\mathrm{Nucl}$. Instrum. Methods Phys. Res. A 784 (2015), pp. 260-263.

[9] H. Harano, T. Matsumoto, Y. Tanimura, Y. Shikaze, M. Baba and T. Nakamura, Monoenergetic and quasi-monoenergetic neutron reference Fields in Japan, Radiat. Meas. 45 (2010), pp. 1076-1082. 